\title{
Risorgimento und Jahrhundertwende. Zur Funktion der Erinnerungskultur in der Nuova Antologia um 1900
}

\author{
SUSANNE FRIEDE
}

Auf welche Weise einzelne Datensätze aus einer Datenbank zu europäischen Kulturzeitschriften der Jahrhundertwende ${ }^{1}$ zu einem umfassenderen Verständnis von nationalen oder internationalen Kulturphänomenen der Jahrhundertwende beitragen können, ist von vielerlei Faktoren abhängig. Ausschlaggebend ist vor allem, welche Kulturzeitschriften im einzelnen betrachtet werden, ob es sich um ein primär national oder primär disziplinär grenzüberschreitendes ${ }^{2}$ Kulturphänomen handelt und welche der in der Datenbank für die grenzüberschreitenden Artikel der Kulturzeitschriften berücksichtigten Kategorien ${ }^{3}$ für die jeweilige Fragestellung vorrangig zu nutzen sind.

Schon aufgrund der Vielfältigkeit der in den Kulturzeitschriften behandelten Disziplinen, Gegenstände und Diskurse ${ }^{4}$ kann nur exemplarisch gezeigt werden, wie sich die von der jeweiligen Kulturzeitschrift gewählte nationale oder transnationale, disziplinär gebundene oder auch gerade diskursiv geöffnete Perspektivierung für das im Einzelfall untersuchte Kulturphänomen darstellt.

1 Siehe unter www.kulturzeitschriften1900.uni-goettingen.de einsehbare Datenbank.

2 Die Leitfrage nach dieser, doppelten Grenzüberschreitung, die in den jeweiligen Kulturzeitschriften und ihren ,Bezugskulturen“ in recht unterschiedlicher Weise sichtbar wird, prägte sowohl die im Rahmen des Göttinger Akademievorhabens aus der Untersuchung der europäischen Kulturzeitschriften entwickelte Datenbank als auch die Ausrichtung der Kolloquien.

3 Dies sind bei grenzüberschreitenden Artikeln (neben einer grundlegenden bibliographischen Erfassung, der Verschlagwortung und Angaben zur Textsorte) die Angabe von Import- und Kontaktländern, die Charakterisierung des Transferguts (nach Gegenständen, Wissenschaften und Realitätsbereichen), die Nennung der beteiligten Kulturträger (getrennt nach Einzelpersonen und Körperschaften, Medien und Institutionen), der Hinweis auf fremdsprachliche Wörter und Wendungen und Besonderheiten sowie ein Resümee. Zu einer exemplarischen Auswertung der Kategorien vgl. den Beitrag: „Il pericolo giallo?“ Der Chinadiskurs in der Nuova Antologia 1899-1901 in diesem Band.

4 Vgl. zur Multidisziplinarität in der Kulturzeitschrift Albrecht/ Friede 2003 und 2004; zu den zentralen (oder auch marginalisierten) Gegenständen und Diskursen siehe außer den Beiträgen in diesem Band auch Europäische Kulturzeitschriften um 1900 als Medien transnationaler und transdisziplinärer Wahrnehmung. 
Eine solche exemplarische Betrachtung führt jedoch auch $\mathrm{zu}$ weitergehenden Aussagen über einzelne Zeitschriftenprofile, über die national oder international vorherrschende Kontextualisierung bestimmter Kulturphänomene oder über die Stellung einzelner Disziplinen innerhalb der Zeitschriften.

Darüber hinaus erlaubt der gewählte Gegenstand des italienischen Risorgimento eine Perspektive, die Ausgangs- und Zielpunkt des Unternehmens über das erinnerungskulturelle Paradigma in Relation zueinander setzt: Die Kulturzeitschrift und die Datenbank sind als zwei mediale Organisationsformen zu verstehen, die über ihre besondere Beziehung zum kontinuitätsstiftenden Paradigma der Erinnerungskultur miteinander verbunden sind.

Die Datenbank wie auch die Kulturzeitschrift lassen sich mit Bezug auf die jüngere kulturwissenschaftliche Gedächtnis- oder Erinnerungsforschung als zwei Formen eines ,kollektiven Gedächtnisses“5 verstehen - oder auch als Formen eines „,Gedächtnisses der Gesellschaft" . ${ }^{6}$ Sie zeugen dabei jedoch von strukturell unterschiedlichen Erinnerungsweisen und Gedächtnissen:

Die Kulturzeitschrift läßt sich metaphorisch als ,Archiv“ beschreiben, in dem Spuren der kollektiven Erinnerung wie in einem Speicher abgelegt sind. Dabei wirkt dieses Archiv-Gedächtnis zugleich als Ordnungsprinzip, das die abgelegten Gegenstände auswählt und anordnet. Gleichzeitig wird es für die Leser auch zum Anlaß für neue Erinnerungsakte. In diesem Zusammenhang darf man sich daher nicht vom äußeren Charakter der Kulturzeitschrift täuschen lassen, der diese auf den ersten Blick eher als einen ,Fundus ${ }^{7}{ }^{7}$ als ein Sammelsurium unterschiedlicher Gegenstände und damit fast beliebig aufgenommener, nur aneinandergereihter Spuren des Erinnerten oder Anlässe zur Erinnerung erscheinen läßt.

Bereits der Zeitschriftentitel „Nuova Antologia“ verweist auf eine positivistisch motivierte Zusammenstellung von Beiträgen und Informationen aus weiten Bereichen der Kultur, aber auch auf die ,Auswahl' des Aufgenommenen. Der Zusatz ,nuova“ und der Untertitel „Lettere, scienze ed arti“ impli-

5 Das „kollektive Gedächtnis“ ist kulturwissenschaftlich mit Rekurs auf Maurice Halbwachs aufgefaßt worden als die „Gesamtheit der [...] Strukturen von materialen Objektivationen vergangener Zeiten, von Erfahrungen und Wissensbeständen und nicht zuletzt: von Formrepertoires einer Gemeinschaft, die als eine Art Fundament den Erinnerungskulturen zugrunde liegt und aus der die einzelnen, der kulturwissenschaftlichen Analyse zugänglichen medial gestützten kollektiven Erinnerungsakte [...] hervorgehen." (siehe Erll 2004, S. 121) Vgl. zu Definition und Bedeutung des „,kollektiven Gedächtnisses“ auch A. Assmann, S. 131 f., sowie Frank/ Rippl, S. 15-

$6 \$ 8$.zuletzt bei Zierold 2006 u. Esposito 2002.

7 Siehe Zierold, S. 130 f., zur Unterscheidung von „Archiv“ und „Fundus“. Vgl. auch Esposito, S. 42 f., zur Unterscheidung einer bloßen Sammlung von Gegenständen in einem Speicher (charakteristisch für die Form des rhetorischen Gedächtnisses) und dem „Archiv“, dem ein Ordnungsprinzip zugrunde liegt (charakteristisch für die Form des Gedächtnisses als Kultur, das mit dem Buchdruck und den Massenmedien einhergeht). Der Leser sieht sich z. B. für die Nuova Antologia mit 6400 Druckseiten pro Erscheinungsjahr konfrontiert. 
zieren dabei die disziplinäre Spannweite, innerhalb deren neu ausgewählt und angeordnet wird. ${ }^{8}$

Im Unterschied zur medialen Organisationsform der Kulturzeitschrift läßt sich die Datenbank metaphorisch als ,Netz' beschreiben, das die durch sie bewahrten Erinnerungen nicht statisch speichert, sondern auf der Basis eigener Operationen bei jeder Anfrage selektiv Gedächtnisbausteine erzeugt. ${ }^{9}$ Die damit seit der Entstehung der Idee des Computers und seiner Vorfahren in den 40er Jahren des 20. Jahrhunderts angelegte „Überwindung des Archivs“ ist vielfach als „Ankunft im Unverdrängten“ (so der Titel eines Aufsatzes von Stephan Porombka) und als digital-architektonische Verwirklichung der „Phantasie vom virtuell vollständigen Gedächtnis“ gefeiert worden, das das „Figurative des Gedankens und die Dynamik der Assoziation“ (Porombka, S. 314) des menschlichen Gedächtnisses einholen könne.

Diese Phantasie erscheint zwar angesichts der Datenbank, die die Basis für den vorliegenden Beitrag bildet, eine weitreichende Utopie; dennoch entscheiden tatsächlich die Programmierung der Datenbankstruktur und die Art der Dateneingabe darüber, welche operationalen Zugriffe von dieser medialen Organisationsform überhaupt ermöglicht werden. Neben der strukturell grundlegend anderen Art des Zugriffs unterscheidet sich die Datenerfassung durch die Datenbank in unserem Fall vor allem durch die gewählte BasisIndexierung ${ }^{10}$ der nationalen oder disziplinären Grenzüberschreitung der durch Datensätze ,abgebildeten“ Artikel von der - letztlich auf die Erstellung von sechs gebundenen Bänden pro Jahr abzielenden - linear reihenden ,Archivierungweise' der Artikel durch die Kulturzeitschrift.

In erinnerungskultureller Perspektive ist in der Nuova Antologia unmittelbar um die Jahrhundertwende (1899-1901) vor allem ein Thema durch Quantität und Varianz der einschlägigen Beiträge hervorzuheben: das Risorgimento, das zentrale Ereignis der italienischen Geschichte des 19. Jahrhunderts. Es wird in der Geschichtswissenschaft inzwischen nicht mehr als überschaubare ,Zeit der Nationalhelden“" (Reinhardt, S. 172), sondern als langandauernde Epoche aufgefaßt, die schon 1796 einsetzte. In der entscheidenden Phase des Einigungsprozesses gibt es darüber hinaus verschiedene absolute Daten, die als Endpunkt der Einigung angesehen werden können: 1861 waren unter Vittorio Emanuele

8 Siehe hierzu Friede/ Albrecht 2004 sowie schon die Einschätzung Guido Biagis im Vorwort zu den 1901 von ihm angefertigten ersten Indici (vgl. Spadolini, S. 37-44).

9 Siehe Weinberg/ Windisch sowie Porombka, vor allem: S. 314: „,[Der Nutzer] steht im Kontakt mit einem Netz, das vorgibt, ihn zu umfangen, und in dem er sich - so heißt es - assoziativ, sprunghaft bewegen kann. Dieses Netz ist an allen Punkten erweiterbar, neue Daten können hineingenommen und mit den bestehenden Daten verknüpft werden. Es sind solche Datenbanken, die heute zunehmend unser Vorstellungsvermögen vom Gedächtnis bestimmen."

10 Diese führt - anders als das ,Archiv “ - bereits strukturell eine ,Bewertung' ein, indem zwischen grenzüberschreitenden und dann ,tiefenerfaßten' und nicht-grenzüberschreitenden und daher bibliographisch erfaßten Artikeln unterschieden wird. 
II. alle Teile Italiens außer Venetien und dem Kirchenstaat vereint; 1866 kam durch den ,Frieden von Wien' Venetien hinzu; 1870 wurde Rom Hauptstadt eines erst durch die Beendigung der päpstlichen Herrschaft über das weltliche Rom völlig geeinten Italien. ${ }^{11}$ Im hier untersuchten Zeitraum lagen die abschließenden Ereignisse des Risorgimento also bereits um die 30 Jahre zurück.

Wie häufig das Risorgimento in der Nuova Antologia in den Jahren um die Jahrhundertwende behandelt wird, erfährt man tatsächlich nicht unbedingt durch eine fortlaufende Lektüre der Kulturzeitschrift, sondern vielmehr durch den operationalen quantitativen und dank der Indexierungen und Resümees auch qualitativen Zugriff der Datenbank. ${ }^{12}$ Zum Zeitpunkt der Abfassung fördert eine Volltextsuche des Begriffs „Risorgimento“ in den für die Nuova Antologia angelegten Datensätzen 71 Treffer zutage.

Die Auswertung dieser Datensätze legt drei Leitfragen nahe, die sich im Hinblick auf die Berichterstattung der Nuova Antologia über das Risorgimento im Kontext der kulturwissenschaftlichen Erinnerungs- und Gedächtnisforschung stellen.

Warum ist das Risorgimento auch 50 Jahre nach den Einheitskämpfen ein so bestimmendes Thema in der Nuova Antologia? Welche disziplinär grenzüberschreitenden Zugriffe und Ordnungsprinzipien werden im ,Archiv ${ }^{\circ}$ der Kulturzeitschrift verwendet, um das Risorgimento für Gedächtnis und Erinnerung verfügbar zu halten? Und schließlich - und dabei ist an dieser Stelle nicht über eine Hypothesenbildung hinauszugelangen -: was sagt dies über das Profil und die Funktionsweise des Mediums aus?

Für die Beantwortung der ersten Frage ist es notwendig, auf Erkenntnisse der Gedächtnisforschung zurückzugreifen. Im Anschluß an die Überlegungen von Aleida und Jan Assmann - auch wenn diese nicht mehr unumstritten sind geht diese davon aus, daß nach einem Zeitraum von drei bis vier Generationen das sogenannte „kommunikative Gedächtnis“, ein „Kurzzeitgedächtnis der Gesellschaft", das Erinnerungen in der Koppelung an einen in interaktiver Praxis erfahrbaren alltagsnahen Erlebniszusammenhang bewahrt und weitergibt, allmählich erlischt. ${ }^{13}$ Das kommunikative Gedächtnis bezieht sich dabei nach Jan Assmann vorrangig auf die „Geschichtserfahrungen im Rahmen individueller Biographien“ (J. Assmann, S. 56) und ist gekennzeichnet durch ein

11 Vgl. Schumann, S. 207-214; Candeloro, S. 9-178, 278-296 und 351-381; sowie Reinhardt, S. 201-232.

12 Weder die im folgenden untersuchten Leitfragen noch überhaupt die Bedeutung des Risorgimento für die Nuova Antologia der Jahrhundertwende wären ohne die Aufbereitung der Artikel in den Datensätzen der Datenbank erkennbar gewesen.

13 Siehe Welzer 2002, S. 14. 
hohes $\mathrm{Ma}$ an Unspezialisiertheit, mangelnde thematische ,Festgelegtheit' und Unorganisiertheit und eine unspezifische Trägerschaft. ${ }^{14}$ Man geht in diesem Zusammenhang auch von einem "Generationengedächtnis“ aus, das als Gedächtnis einer Altersgruppe ,geprägt [wird] durch herausragende historische Ereignisse, durch Sprech- und Denkweisen, durch Vorbilder und Mitmenschen, durch Utopien und Traumata. Dieses Gedächtnis lebt, solange die Angehörigen einer Generation leben, es ist unmittelbar mit seinen Trägern, den Zeitzeugen einer Erfahrungs- und Erinnerungsgemeinschaft, verbunden und reicht in der Regel nicht weiter zurück als etwa 80 Jahre." (Keppler, S. 142) ${ }^{15}$

Unabhängig von der Möglichkeit einer genauen Bestimmung der zeitlichen Reichweite des kommunikativen Gedächtnisses ist festzustellen, daß das an Individuen gebundene Erfahrungsgedächtnis bezüglich der Einigungskämp$\mathrm{fe}$, das Generationengedächtnis der Gruppe der Einheitskämpfer, in den Jahren 1899 oder 1900 im Aussterben begriffen ist. Bei einer niedriger als heute anzusetzenden Lebenserwartung war in Italien allmählich fast niemand mehr am Leben, der 1859 oder 1860 oder gar im Vorfeld noch aktiv mitgekämpft oder die politischen Prozesse der 1850er Jahre mitgestaltet hatte. So verstummte diese Möglichkeit des kommunikativen Austausches über und des interaktiven Zugriffes auf die Vergangenheit zunehmend. Wenn jedoch ein erinnertes Ereignis so wichtig für die erinnernde Kultur - vertreten durch mehr oder weniger große gesellschaftliche Gruppen - ist oder erneut wird, daß es nicht dem Vergessen anheimfallen darf, sind die Voraussetzungen für einen Aufschwung der Erinnerungskultur gegeben.

Auf diese Weise entsteht offenbar - wie die Berichterstattung der Nuova Antologia erkennen läßt - im Falle des Risorgimento mit dem Erlöschen des kommunikativen Gedächtnisses allmählich das Bedürfnis nach einem anderen ,Gedächtnis-Rahmen“, dem sogenannten „,kulturellen Gedächtnis“, das durch die Organisiertheit einer spezifischen Trägerschaft, die Verbindlichkeit im Bezug auf das normative Selbstbild einer Gruppe und durch die Reflexivität der gängigen Praxis in der Lebensperspektive der Gruppe bestimmte selektive Gedächtnisbausteine verwaltet und rituell reproduziert. ${ }^{16}$ Das Risorgimento brachte als ,Umbruchsereignis“ ohnehin eine noch lange nachwirkende Krise von Erklärungs- und Interpretationsmustern hervor, die jedoch auch ein gesteigertes Reflexionspotential über die Gesellschaft und die Deutung ihrer Vergangenheit freisetzte.

Aus den auf das Risorgimento bezogenen Artikeln, die um die Jahrhundertwende in der Nuova Antologia erschienen, läßt sich auf eine Umbruchsphase des Übergangs vom kommunikativen - sicher jedenfalls vom Erfahrungsgedächtnis - zu der Organisationsform eines erweiterten ,Gedächtnis-Rahmens`

14 Vgl. J. Assmann, S. 48 ff., Welzer 2002, S. 13 ff.; Pethes, S. 59 ff.; Zierold, S. 68 f.

15 Siehe Pethes, S. 61 f., zum Verhältnis von biographischem und Generationengedächtnis.

16 Siehe Erll 2005, S. 27-29; A. Assmann, S. 56; Zierold, S. 70-73. 
schließen. Ohne daß man für den kleinen zeitlichen Ausschnitt von drei Jahren, den die Datenbank verzeichnet, bereits von der Etablierung eines ,kulturellen Gedächtnisses' sprechen kann, wird jedenfalls in der Kulturzeitschrift das dringende Bedürfnis erkennbar, das vom Verschwinden bedrohte kommunikative Gedächtnis fortzuschreiben.

II.

Dies führt zur zweiten Frage nach den Ordnungsprinzipien, die im Archiv der Kulturzeitschrift angewandt werden, um das Risorgimento für Gedächtnis und Erinnerung verfügbar zu halten.

Bei der Archivierung sind beide Modi von Retention und Konstruktion, die dem kulturellen Gedächtnis allgemein zugeschrieben werden, gleichermaBen erkennbar: der als Speichergedächtnis wie auch der als Funktionsgedächtnis. ${ }^{17}$ Der Modus des Speichergedächtnisses gewährleistet die Potentialität der Erinnerung und sorgt für die Unantastbarkeit als wichtig erachteter Texte und die Bewahrung des autonomen Status von individuellen Dokumenten. Hiervon zeugen in der Nuova Antologia diejenigen Artikel, die vorwiegend Dokumentationscharakter haben. Sie verweisen auf die Position der Kulturzeitschrift an der Schnittstelle zwischen der Dokumentation des kollektiven Gedächtnisses und einer historischen Darlegung der Ereignisgeschichte im engeren Sinne.

In solchen Artikeln werden bisher nicht gedruckte Dokumente vorgelegt und erörtert. Sie zeugen einerseits von einem positivistischen Streben zur lückenlosen Geschichtsdarstellung, andererseits von einer damit verbundenen Monumentalisierung der Erinnerung. Beide Aspekte finden sich in Fortsetzungsartikeln wie „Sulla via di Roma“"18 und in Artikeln zu einzelnen zentralen politischen Ereignissen des Risorgimento, z. B. zur „Convenzione del 15 Settembre 1864" "19. Immer wieder wird auf Cavour und Garibaldi referiert. ${ }^{20}$ Auch die unmittelbar vor der Zuspitzung des Einigungsprozesses liegende Vergangenheit wird in einigen Artikeln aufgearbeitet. Hierunter fällt beispielsweise das Wirken der österreichischen Regierung in Mailand von 1828 bis 1836. ${ }^{21}$ Solche Fortschreibungen der Erinnerung in die Vergangenheit müssen eindeutig jenseits des kommunikativen Gedächtnisses verortet werden, wodurch der fließende Übergang zwischen dem Fortschreiben eines kommunikativen und der von der Kulturzeitschrift vorangetriebenen allmählichen

17 Siehe zu den beiden Modi A. Assmann, S. 133-142; Welzer 2002, S. 14.

18 Siehe u. a. die drei Artikel der Fortsetzung Sulla via di Roma aus dem Jahr 1900.

19 Siehe u. a. den Artikel von einem nicht namentlich zeichnenden Autor (Un po' più di luce sulla Convenzione del 15 Settembre 1864) sowie von Marco Minghetti aus dem Jahr 1899.

20 Siehe u. a. die Artikel von Bertolini und De Amicis aus dem Jahr 1900.

21 Siehe u. a. die Artikel von Alessandro D'Ancona aus dem Jahr 1899. 
,Archivierung“ von Bausteinen eines „kulturellen Gedächtnis“ besonders deutlich wird.

Entscheidend sind für den Speichermodus des solchermaßen fortgeschriebenen kommunikativen Gedächtnisses also zum einen die Unantastbarkeit von implizit als ,ewig gültig' ausgewiesenen Dokumenten, zum anderen die Rückbindung an historisch zentrale Figuren wie Cavour und Garibaldi. Daneben wird jedoch vor allem auch die ,zweite' oder, dritte' Reihe der unbekannteren Helden der Vereinigung oder ihrer Vorreiter aufgearbeitet. Einschlägig ist ein Artikel vom Januar 1899 aus der Rubrik Notizia letteraria, der den Titel „Per la storia del risorgimento italiano" trägt. ${ }^{22}$ Es werden die neu veröffentlichten Memoiren Angelo Frignanis, Giacomo Comandinis und Aurelio Saffis sowie eine Studie zu Pellegrino Rossi rezensiert. Alle Genannten sind dem Zielpublikum wohl zumindest dem Namen nach bekannt gewesen; wie detailliert die Kenntnisse bezüglich solcher Akteure jedoch beim Zielpublikum der Nuova Antologia im einzelnen gewesen sind, ist nicht mit Sicherheit festzustellen. Deutlich erkennbar ist das Ziel der Kulturzeitschrift, für die italienische Gesellschaft, zumindest für eine bestimmte Gruppe, den Zugriff auf das Individualund Familiengedächtnis auch weniger herausragender historischer Persönlichkeiten des Einigungsprozesses offenzuhalten und so die Fortschreibung des kommunikativen Gedächtnisses über die Reichweite des Generationengedächtnisses hinaus zugänglich zu halten. ${ }^{23}$

Die Aufgabe des kulturellen Gedächtnisses als Speichergedächtnis wird gleichberechtigt durch dessen Modus als Funktionsgedächtnis ergänzt: Dieser Modus dient der jeweils aktualisierten Fundierung der Gegenwart auf einer durch die Struktur des kulturellen Gedächtnisses ausgewählten und konstruierten - Vergangenheit. Dabei herrscht ein selektiver und strategisch ausgerichteter Gebrauch der Erinnerungen vor, der so der Profilierung einer distinktiven Gruppenidentität dienen kann. Diese Feststellung kommt neuesten Theorien zur „,kollektiven Erinnerung“ entgegen, denen zufolge der Gedächtnisbegriff durch den der ,gesellschaftlichen Erinnerung“, jedenfalls durch die vorherrschende Vorstellung von „Erinnerungszugriffen“, ersetzt werden soll. ${ }^{24}$

Entscheidend ist für die diesem Modus entsprechende Perspektivierung der Nuova Antologia, daß der funktionale Zugriff auf das Risorgimento vor allem auf dessen Funktion als Gründungsmythos rekurriert. Die Verfasser der Artikel betonen, daß eine ständige aktuelle Verinnerlichung der durch das Risorgimento geschaffenen Bedingungen unabdingbare Voraussetzung dafür sei, die gegenwärtige gesellschaftliche und politische Situation in Italien, ja überhaupt jede Situation zwischen 1848 und $1900 \mathrm{zu}$ verstehen. Meistenteils fungiert die Er-

22 Siehe den Artikel von Ernesto Masi aus dem Jahr 1899.

23 Vgl. zu Vorreitern im Einigungsprozeß u. a. die Artikel von Carlo Osvaldo Pagani und Domenico Zanichelli aus dem Jahr 1900.

24 Siehe besonders Zierold, passim (S. 42 f. zur Auffassung des Gedächtnisses als bloßer Funktion; S. 111 ff. zu Erinnerung und „Konnektivität“). 
wähnung des Risorgimento dabei wie der Topos von einem in vieler Hinsicht goldenen Zeitalter, auf das die aktuelle Situation der italienischen Gesellschaft vielfailtig zurückbezogen werden kann und muß. Er wird mit mahnenden Rückbezügen auf die Außen-, Innen- und sogar auf die Finanzpolitik der Jahre 1899 und 1900 angewandt. Nicht nur Diskussionen um den Status der Monarchie und des Papstes, sondern auch solche um militärische Niederlagen in den afrikanischen Gebieten werden auf das Risorgimento rückbezogen. ${ }^{25}$

Denjenigen Artikeln, die dem Funktionsgedächtnis zuzuordnen sind, liegt dabei als Subtext meist zugrunde, daß es gilt, nationalen Dekadenzerscheinungen Einhalt zu gebieten und den Idealzustand des Risorgimento in der italienischen Mentalität wiederzubeleben. Stellenweise wird in diesem Zusammenhang sogar der Ruf nach einem neuen Cavour laut. Ein funktional erinnernder Zugriff auf das Risorgimento wird besonders in einem Artikel Edoardo Arbibs vom August 1900 deutlich, der den Titel „L'ideale della patria“ trägt. Der Verfasser deutet nicht nur die Ermordung Umbertos I. im Juli 1900 als Ausdruck eines allerorten sichtbar werdenden moralischen Verfalls in der italienischen Gesellschaft - und damit als eigentliches Ende des Risorgimento -, sondern führt alle Dekadenzerscheinungen auf den Verlust der risorgimentalen Selbstsicherheit zurück, die 1859 dominiert habe, im Jahre 1900 jedoch verschwunden, in erinnerungskultureller Perspektive also: vergessen sei. Um den Geist des Risorgimento dennoch wieder heraufzubeschwören, werden lange Passagen aus privaten Briefen und Dokumenten der Freiheitskämpfer ,der zweiten Reihe' zitiert. Als Ideale dieser Männer werden die unbedingte Liebe zu Italien und die Interesselosigkeit bei der Verwaltung der ihnen anvertrauten öffentlichen Güter gelobt. Abschließend fordert Arbib, daß Vaterlandsliebe und Vertrauen in die Zukunft neu erstehen müßten.

III.

Für die Frage nach einer Erklärung für die Dominanz des erinnerungskulturellen Bezugs auf das Risorgimento in den untersuchten Jahrgängen ist die Feststellung interessant, daß dieser Bezug fast unmittelbar nach der Jahrhundertwende quantitativ (und damit natürlich auch qualitativ) deutlich zurückgeht. ${ }^{26}$ Offenbar muß im Italien der Jahrhundertwende - weil die Epochenschwelle als Herausforderungslage empfunden wurde - eine erneute Umbruchssituation diag-

25 Siehe u. a. die Artikel von Giulio Alessio (1899), Achille Fazzari (1900) und L.R. (n.n.; 1900).

26 Diese Feststellung ist aus einer extensiven Lektüre der Jahrgänge 1899 bis 1909 der Kulturzeitschrift erwachsen. Diesem Zusammenhang wird in Kürze eine umfangreiche Untersuchung gewidmet sein. Signifikant ist jedoch, daß bereits im Jahr 1901 viel weniger einschlägige Artikel zu verzeichnen sind (von denen hier aus thematischen Gründen keiner behandelt wurde). 
nostiziert werden, in der wiederum ein Reflexionspotential über die Gesellschaft freigesetzt wird, die sich vor allem des Stellenwertes der eigenen Geschichte und durch eine aktualisierende Deutung des Vergangenen ihrer selbst vergewissert. In dem Moment, in dem die Epochenschwelle jedoch, sicher überschritten' ist, wird eine Selbstvergewisserung der Gruppe mit Bezug auf ihren Gründungsmythos offenbar weniger wichtig, und statt dessen rücken die Zukunft und ihre Beschleunigungsprozesse in den Vordergrund - nicht umsonst dominiert seit 1899 auch, und dies über die Jahrhundertwende hinaus, die Berichterstattung über die Neuverwaltung und den Ausbau des italienischen Eisenbahnwesens.

Die Frage, was die aus den Datensätzen gewonnenen Erkenntnisse über das Profil und die Funktionsweise der Nuova Antologia aussagen, paßt durchaus zur jüngst erfolgenden Fokussierung der kulturwissenschaftlichen Gedächtnis- und Erinnerungsforschung auf die Rolle der Medien für Erinnerungsprozesse. Entsprechende Untersuchungen zur Relation von Medien und Erinnerungsprozessen weisen darauf hin, daß, obwohl offenbar keine medienspezifischen Gedächtnisformen auszumachen sind, das Gedächtnis der Gesellschaft und die Medien phänomenologisch nicht zu entkoppeln seien. ${ }^{27}$ Wenn nicht schon mit Jan Assmans Schrift zum Kulturellen Gedächtnis, so ist es mit Elena Espositos Untersuchung zum Sozialen Vergessen Konsens geworden, daß es eine mediengeschichtlich bedingte Veränderung unserer Wahrnehmung gibt.

Vielleicht nicht aufgrund der nicht sicher zu bestimmenden Auflage, aber auf der Basis einer stabilen Abonnentengruppe - und gewiß vom Anspruch her - ist die Nuova Antologia ein weitreichendes Medium, wenn auch kein Massenmedium. ${ }^{28}$ Wie die Massenmedien ist sie systematisch jedoch auch dadurch zu bestimmen, daß sie als gesellschaftliche Funktion die Erzeugung eines medialisierten Gedächtnisses zur Aufgabe hat. ${ }^{29}$ Jedes kollektive und damit soziale Gedächtnis ist - wie zuerst Maurice Halbwachs in La mémoire collective (1939) formulierte - gruppengebunden, und so auch das von der Kulturzeitschrift repräsentierte. Im Falle der Nuova Antologia wird die ,Gedächtniserzeugung، angesichts der nationalen Reichweite der Zeitschrift durch eine erstaunlich überschaubare Gruppe von Produzenten geleistet. Sie wird angeführt und ge-

27 Siehe Zierold, passim u. Esposito, S. 38: „Die Leithypothese dieser Arbeit besteht in der Annahme, dass im Verlauf gesellschaftlicher Evolution zwischen den Differenzierungsformen der Gesellschaft und den Kommunikationstechnologien ein gegenseitiger Anpassungsdruck und eine ständige Wechselwirkung herrschen. Das Gedächtnis der Gesellschaft ist jeweils das Ergebnis dieser Dynamik." (Vgl. aber S. 39 f. gegen die Annahme z. B. eines „Fernsehgedächtnisses“.)

28 Vgl. Friede u. die Primärtexte in Spadolini.

29 Siehe Zierold, S. 189-199; Esposito, S. 263. Die Massenmedien vertreten nach Esposito eine „Kommunikation, die nicht auf Überzeugungen [...] gründet, sondern lediglich auf der Annahme, dass man informiert sei [...]. Was geteilt wird, sind also lediglich Informationen, die insofern das Gedächtnis konstituieren, als sie erinnert werden müssen, weil sie dazu erforderlich sind, neue Informationen zu verstehen.“ 
prägt durch die zentrale Rolle des Eigentümers und Direktors Maggiorino Ferraris, der für die Auswahl der Beiträger und auch die Einwerbung und Beurteilung der Artikel verantwortlich war. ${ }^{30}$ Die in der Nuova Antologia veröffentlichenden Autoren setzten sich zu annähernd gleichen Teilen aus Wissenschaftlern, Politikern oder Militärs und Kunstkritikern oder Literaten zusammen. ${ }^{31}$ Für fast jedes behandelte Gebiet etablierten sich bestimmte Autoren als Experten: Für das Risorgimento sind dies in den untersuchten Jahren z. B. Edoardo Arbib sowie der nicht namentlich zeichnende Autor, der die Fortsetzungen „Sulla via di Roma“ verfaßte. ${ }^{32}$ Das Prestige der Autoren wird dabei auch durch die konstante Nennung der Titel demonstriert ${ }^{33}$ so ließ sich Ferraris selbst in der Kulturzeitschrift stets mit dem ,Titel' als Abgeordneter (,onorevole") bezeichnen.

Die Kulturzeitschrift erzeugt so Kommunikationsakten der kollektiven Erinnerung ihrer Produzenten- und Zielgruppe und bezeugt Prozeduren der Übermittlung eines kollektiven Gedächtnisses. Dies kann im einzelnen sowohl intentional als auch unwillkürlich abbildend geschehen. Die mit dem Medium verbundene Gruppe nutzt die Kulturzeitschrift sowohl auf der Produktions- als auch auf der Rezeptionsseite als soziale Praktik, um ihre Vorstellung von der Vergangenheit $\mathrm{zu}$ vermitteln und zu bewahren. So konstituiert sich über das Medium eine ,Erinnerungsgemeinschaft ${ }^{*}{ }^{34}$

Die Nuova Antologia ist dabei als das ausdifferenziert, was ein Elitemedium genannt werden kann. Sie führt gruppenspezifische, aber vom Anspruch her hegemonial wirkende verbindliche Konsensverpflichtungen ein. ${ }^{35}$ Die soziale Konstruktion von Erinnerung, gerade der an das Risorgimento, tritt so deutlich zutage. Die Kulturzeitschrift entwirft und pflegt ein fortgeschriebenes und fortzuschreibendes kommunikatives Gedächtnis des Risorgimento, das der Einübung von Handlungs- und Bewertungsschemata dient, und produziert bestimmte kulturelle Wahrnehmungsmuster, z. B. dasjenige von der wünschenswerten überzeitlichen Orientierung der Italiener an der Mentalität der Einigungskämpfer.

30 Gegen Ferraris‘ Votum konnte nichts entschieden werden. Lediglich im literarischen und künstlerischen Ressort wurde er durch den Chefredakteur Giovanni Cena unterstützt. Vgl. Ricorda, S. 77-86.

31 Siehe hierzu Friede, S. 36 f.

32 Vgl. ähnliches auch für den Chinadiskurs, der ebenfalls in diesem Band behandelt wird.

33 Siehe Barner, S. 49; Friede, S. 36.

34 Für den Terminus der Erinnerungsgemeinschaft siehe Lottes, S. 175. Nach Lottes ist dabei ein gemeinsames soziales Milieu entscheidend, das für die Produzenten und Leser der Nuova Antologia in der Tat vorausgesetzt werden kann. Vgl. auch Keppler, S. 144 f. (zur sozialen Praktik und den Prozeduren der Übermittlung).

35 Zum Verhältnis von kulturellem Gedächtnis und Hegemonieanspruch vgl. Erll 2005, S. 119. 
Das Risorgimento ist in diesem Zusammenhang eine durch das Medium gestiftete ,Leiterinnerung ${ }^{6}{ }^{36}$ die sowohl als Schlüsselkategorie des Vergesellschaftungsprozesses im postunitarischen Italien als auch als Zugriffsmöglichkeit für die Identitätsgeschichten der Leser fungiert. Damit bringt die Nuova Antologia jedoch nur eine von mehreren möglichen, auch parallel existierenden, Erinnerungskulturen zum Ausdruck. Ihre ,Kommunikationsakten' sind dabei nicht Teil der Geschichtswissenschaft, sie beschreiben jedoch willkürlich und unwillkürlich Geschichte. ${ }^{37}$

Die Nuova Antologia stärkt und selektiert durch die Berichterstattung bestimmte Erinnerungsbausteine, die sich in einen allgemeingesellschaftlichen Verfestigungs- und Kanonisierungsprozeß zum Risorgimento einfuggen, von dem auch zahlreiche Hinweise in den Rubriken Tra libri e riviste und Notizie, Libri e recenti pubblicazioni (auf Festreden oder neu errichtete Monumente) zeugen. Die Berichterstattung der Kulturzeitschrift trägt daher wesentlich zur Auswahl und Verfestigung von lieux de mémoire ${ }^{38}$ im Sinne Pierre Noras bei.

Für das Profil und die Funktionsweise der Nuova Antologia läßt sich daher durch die Auswertung der auf das Risorgimento bezogenen Datensätze festhalten:

Die Erinnerung an das Risorgimento dient um das Jahr 1900 der Identitätsstiftung einer sozial und mental zu diesem Zeitpunkt immer noch wenig homogenen Gemeinschaft, die sich als ,Erinnerungsgemeinschaft' einer Nation erst konstituieren muß. Gerade die Veröffentlichung von Individualerinnerungen bietet dabei gruppenverstärkende Erinnerungsanlässe. Die Gruppe der Produzenten steuert dabei, stellvertretend für die Zielgruppe der ebenfalls in der Gesellschaft agierenden Leser, die Identitätsbildung der Gruppe aus dem Bezug auf die risorgimentale Vergangenheit. Interessant ist hierbei, daß eine Abfrage des Begriffs in den Datensätzen der untersuchten französischen und deutschen Kulturzeitschriften keinen einzigen Treffer ergibt. Daraus läßt sich bei aller gebotenen Vorsicht schließen, daß die historische Situation des ,jungen Italien' bei alteingesessenen europäischen Nationen um die Jahrhundertwende nur ein sehr geringes Interesse fand. ${ }^{39}$ Das Risorgimento übernimmt also - wie die Kulturzeitschrift zeigt - nur für den nationalen italienischen Kontext integrativ die Rolle einer positiv bewerteten Leiterinnerung, differenziert diese

36 Siehe Lottes, S. $178 \mathrm{f}$.

37 Die in der Datenbank verzeichnete Unterscheidung von „Wissenschaften“ und „Realitätsbereichen“ gewinnt hier besondere Bedeutung. Während andere Gegenstände in der Nuova Antologia in der Regel sowohl (wenn auch seltener) populärwissenschaftlich als auch mit dem Fokus auf die Relation von Kulturphänomenen und alltäglicher Realität behandelt werden, gilt dies nicht für die Geschichte.

38 Siehe zu Noras weitem Verständnis des lieu de mémoire Erll 2005, S. 23-27; Pethes, S. 89-93.

39 Vgl. jedoch kontrastiv das relativ große Interesse im China der Jahrhundertwende (siehe den entsprechenden Beitrag in diesem Band). 
jedoch in der disziplinären Grenzüberschreitung weit aus, wobei erklärende, argumentative, mahnende, nostalgische oder therapeutische Zwecke verfolgt werden.

Die Erinnerungsprozesse dienen im hochkulturellen Kontext des Elitemediums auch der Legitimation einer herrschenden Gruppe. Sie verfolgt die Fortschreibung eines kommunikativen Gedächtnisses durch die allmähliche Installation von Erinnerungsbausteinen, die die Basis für eine mögliche Verankerung im kulturellen Gedächtnis einer gruppenübergreifenden Gemeinschaft bilden. So kann letztlich durch die Selbstbeobachtungsfunktion, die die Erinnerungsgemeinschaft für die Gesellschaft übernimmt, auch der mangelnden Orientierungsgewißheit der Gruppe begegnet werden. Dabei ist das Medium sowohl materieller Träger der Erinnerung wie auch ,Erzeuger' immer wieder aktualisierter Erinnerungsbezüge.

Wenn man - zumal für die Zeit vor der Entwicklung der digitalen Medien - „Kultur als Gedächtnis“40 auffaßt, ist die Kulturzeitschrift sicherlich als eine „Gedächtniszeitschrift“ oder genauer: ,erinnerungskulturelle Zeitschrift“ anzusehen. Eine Datenbank des 21. Jahrhunderts kann zwar diese erinnerungskulturelle Funktion der Kulturzeitschrift neu sichtbar machen und hervorheben, inwieweit sie sie aber dauerhaft wird bewahren können, ${ }^{41}$ wird die medientechnische Entwicklung der Zukunft zeigen.

\section{Anhang}

[Aufgenommen sind nur die im Beitrag behandelten Artikel.]

Nuova Antologia: 1899

163 (1899) 193-215 - Alessandro D’Ancona: Spigolature nell'archivio della polizia austriaca di Milano. I. Manzoni-Stendhal [fortgesetzt in 163, 581-593: II.GiobertiCavour].

163 (1899) 261-277 - Ernesto Masi: Per la storia del risorgimento italiano. Notizia letteraria.

163 (1899) 546-565 - Giulio Alessio: Parlamento e Riforme.

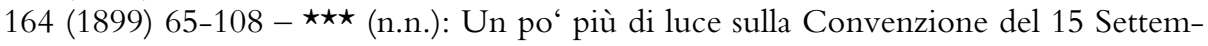
bre 1864 .

165 (1899) 136-172 - Marco Minghetti: La Convenzione di Settembre 1864.

40 Siehe Esposito 2002, 183 ff., die die dritte Evolutionsstufe des Gedächtnisses der Gesellschaft mit Beginn der Moderne mit „Kultur als Gedächtnis“ bezeichnet.

41 Siehe die Zusammenfassung der kritischen Diskussion bei Zierold, S. 166 ff.: ,Neue“ Medientechnologien: Ende der Erinnerung? 


\section{Nuova Antologia: 1900}

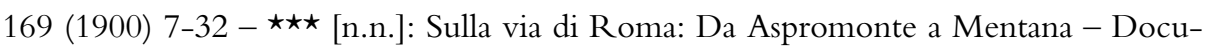
menti inediti - I. [fortgesetzt in 171, 593-610: II. Mentana (1867)].

169 (1900) 674-702 - Edoardo Arbib: Sulla via di Roma: La Questione in Parlamento.

169 (1900) 703-728 - Edmondo De Amicis: Per Giuseppe Garibaldi.

171 (1900) 335-348 - Achille Fazzari: La Costituente.

171 (1900) 672-691 - L.R.: Per la storia del parlamento italiano.

171 (1900) 696-709 - Domenico Zanichelli: La Signora Emilia Peruzzi.

172 (1900) 577-590 - Francesco Bertolini: L'opera di Camillo Benso di Cavour.

172 (1900) 616-631 - Edoardo Arbib: L'ideale della patria.

173 (1900) 452-467 - Carlo Osvaldo Pagani: Per Nicola Marselli nel primo anniversario della sua morte.

\section{Literaturverzeichnis}

Albrecht, Andrea/ Friede, Susanne: Trans-, Inter- und Supradisziplinarität um 1900. Zum Diskurs in deutschen und französischen Kulturzeitschriften, in: Brand, Frank/ Schaller, Franz / Völker, Harald (Hgg.): Transdisziplinarität. Bestandsaufnahme und Perspektiven. Beiträge zur THESIS-Arbeitstagung im Oktober 2003 in Göttingen, Göttingen (Universitätsverlag) 2004, 97-114.

Albrecht, Andrea/ Friede, Susanne: Jahrhundertwende - Literatur, Künste, Wissenschaften um 1900 in grenzüberschreitender Wahrnehmung, in: Newsletter Moderne. Zeitschrift des Spezialforschungsbereichs „Moderne - Wien und Zentraleuropa um 1900“, 7 (2004), Heft 1, 26-29.

Assmann, Aleida: Erinnerungsräume. Formen und Wandlungen des kulturellen Gedächtnisses, München (Beck) 1999.

Assmann, Jan: Das kulturelle Gedächtnis. Schrift, Erinnerung und politische Identität in frühen Hochkulturen, München (Beck) 1999 (Beck'sche Reihe, 1307) [zuerst München 1992].

Barner, Wilfried: Klassikerpflege als innereuropäische Konkurrenz um 1900, in: Europäische Kulturzeitschriften um 1900 als Medien transnationaler und transdisziplinärer Wahrnehmung, 145-154.

Candeloro, Giorgio: Storia dell'Italia moderna, Bd. 5: La costruzione dello Stato unitario, Mailand (Feltrinelli) 1968.

Esposito, Elena: Soziales Vergessen. Formen und Medien des Gedächtnisses der Gesellschaft. Aus dem Italienischen von Alessandra Corti, Frankfurt a.M. (Suhrkamp) 2002.

Erll, Astrid: Erinnerungshistorische Literaturwissenschaft, in: Nünning, Ansgar/ Sommer, Roy (Hgg.): Kulturwissenschaftliche Literaturwissenschaft, Tübingen (Narr) 2004, 115-128.

Erll, Astrid: Kollektives Gedächtnis und Erinnerungskulturen. Eine Einführung, Stuttgart/ Weimar (Metzler) 2005.

Europäische Kulturzeitschriften um 1900 als Medien transnationaler und transdisziplinärer Wahrnehmung. In Zusammenarbeit mit Susanne Friede hg. von Ulrich Mölk, Göt- 
tingen (Vandenhoeck \& Ruprecht) 2005 (Abhandlungen der Akademie der Wissenschaften zu Göttingen, Philologisch-Historische Klasse, Dritte Folge, Band 273).

Frank, Michael C./ Rippl, Gabriele (Hgg.): Arbeit am Gedächtnis. Für Aleida Assmann, München (Fink) 2007.

Friede, Susanne: Europäische Literaturströmungen in der Nuova Antologia um 1900, in: Europäische Kulturzeitschriften um 1900 als Medien transnationaler und transdisziplinärer Wahrnehmung, 33-54.

Keppler, Angela: Soziale Formen individuellen Erinnerns. Die kommunikative Tradierung von (Familien-)Geschichte, in: Welzer, Harald (Hg.): Das soziale Gedächtnis. Geschichte, Erinnerung, Tradierung, Hamburg (Hamburger Edition) 2001, 137159.

Lottes, Günther: Erinnerungskulturen zwischen Psychologie und Kulturwissenschaft, in: Oesterle, Günter (Hg.): Erinnerung, Gedächtnis, Wissen. Studien zur kulturwissenschaftlichen Gedächtnisforschung, Göttingen (Vandenhoeck \& Ruprecht) 2005 (Formen der Erinnerung, 26), 163-184.

Medien des Gedächtnisses. Herausgegeben von Aleida Assmann, Manfred Weinberg, Martin Windisch, Stuttgart/ Weimar (Metzler) 1998 (Deutsche Vierteljahresschrift für Literaturwissenschaft und Geistesgeschichte, Sonderheft).

Pethes, Nicolas: Kulturwissenschaftliche Gedächtnistheorien zur Einführung, Hamburg (Junius) 2008.

Porombka, Stephan: Ankunft im Unverdrängten. Datenbanken als Verkörperung der Phantasie vom virtuell vollständigen Gedächtnis, in: Medien des Gedächtnisses, 313328.

Reinhardt, Volker: Geschichte Italiens, München (Beck) 2003.

Ricorda, Ricciarda: La „Nuova Antologia“ 1866-1915. Letteratura e ideologia tra Ottocento e Novecento, Padova, Liviana, 1980.

Schumann, Reinhold: Geschichte Italiens, Stuttgart et al. (Kohlhammer) 1983.

Spadolini, Giovanni: La Nuova Antologia dal risorgimento alla repubblica (1866-1988). Programmi et bilanci, Florenz (Fondazione Nuova Antologia) 1988 (Estratti della Nuova Antologia, 5).

Weinberg, Manfred/ Windisch, Martin: Einleitung, in: Medien des Gedächtnisses, 1-13.

Welzer, Harald: Das kommunikative Gedächtnis. Eine Theorie der Erinnerung, München (Beck) 2002.

Zierold, Martin: Gesellschaftliche Erinnerung. Eine medienkulturwissenschaftliche Perspektive, Berlin/ New York (de Gruyter) 2006 (Media and Cultural Memory/ Medien und kulturelle Erinnerung, 5). 